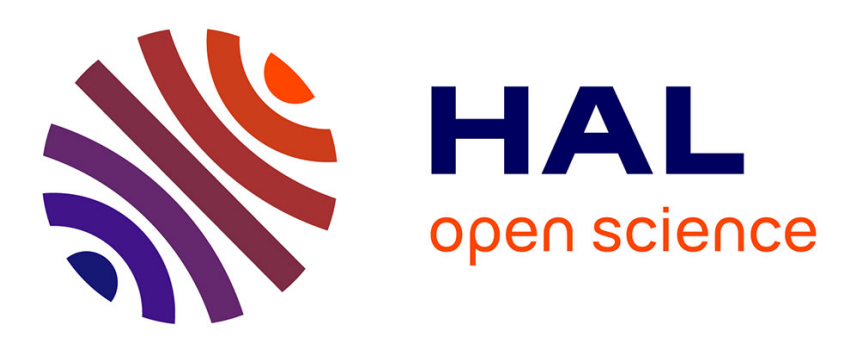

\title{
Frequency doubling and parametric fluorescence in a four-port aluminum gallium arsenide photonic chip
}

Iännis Roland, Adrien Borne, Marco Ravaro, Romain de Oliveira, Stéphan

Suffit, Pascal Filloux, A. Lemaître, Ivan Favero, Giuseppe Leo

\section{- To cite this version:}

Iännis Roland, Adrien Borne, Marco Ravaro, Romain de Oliveira, Stéphan Suffit, et al.. Frequency doubling and parametric fluorescence in a four-port aluminum gallium arsenide photonic chip. Optics Letters, 2020, 45 (10), pp.2878-2881. 10.1364/OL.392417 • hal-03052481

\section{HAL Id: hal-03052481 https://hal.science/hal-03052481}

Submitted on 10 Dec 2020

HAL is a multi-disciplinary open access archive for the deposit and dissemination of scientific research documents, whether they are published or not. The documents may come from teaching and research institutions in France or abroad, or from public or private research centers.
L'archive ouverte pluridisciplinaire HAL, est destinée au dépôt et à la diffusion de documents scientifiques de niveau recherche, publiés ou non, émanant des établissements d'enseignement et de recherche français ou étrangers, des laboratoires publics ou privés. 


\title{
Frequency doubling and parametric fluorescence in a four-port Aluminum Gallium Arsenide photonic chip
}

\author{
IÄnnis Roland ${ }^{1, *}$, Adrien Borne ${ }^{1}$, Marco Ravaro $^{1}$, Romain De Oliveira ${ }^{1}$, Stéphan Suffit ${ }^{1}$, \\ Pascal Filloux ${ }^{1}$, Aristide Lemaître ${ }^{2}$, IVAN Favero ${ }^{1}$, And Giuseppe Leo ${ }^{1}$ \\ ${ }^{1}$ Matériaux et Phénomènes Quantiques, Université de Paris \& CNRS, 10 rue A. Domon et L. Duquet, 75013 Paris, France \\ ${ }^{2}$ Centre de Nanosciences et de Nanotechnologies, CNRS, Univ. Paris-Sud, Université Paris-Saclay 91460, Marcoussis, France \\ *Corresponding author: iannis.roland@univ-paris-diderot.fr
}

Compiled March 7, 2020

\begin{abstract}
We report on the fabrication and characterization of a monolithic III-V semiconductor photonic chip designed to perform nonlinear parametric optical processes for frequency conversion and non-classical state generation. This chip co-integrates an AlGaAs microdisk evanescently coupled to two distinct suspended waveguides designed for light injection and collection around $1600 \mathrm{~nm}$ and $800 \mathrm{~nm}$. Quasi-phase matching provided by the resonator geometry and material symmetry, resonant field enhancement and confinement ensure efficient nonlinear interactions. We demonstrate second-harmonic generation efficiency of $5 \% \mathrm{~W}^{-1}$, and a biphoton generation rate of $1.2 \mathrm{kHz} / \mathrm{\mu W}$ through spontaneous down-conversion. (๑2020 Optical Society of America
\end{abstract}

OCIS codes: (190.4390) Nonlinear optics, integrated optics; (230.4320) Nonlinear optical devices; (220.4241) Nanostructure fabrication.

\section{http://dx.doi.org/10.1364/ao.XX.XXXXXX}

Optical microdisks and microrings, combining high quality factors [1] and micrometer size to enhance the light-matter interaction, are used in an increasing number of applications like microlaser [2], cavity quantum electrodynamics [3], optomechanics [4], biosensors [5], integrated optics [6] and nonlinear optics [7]. While silicon benefits from the huge fabrication knowledge developed for the CMOS industry, its centrosymmetry make nonlinear photonics applications only possible through thirdorder processes and limited by two-photon absorption in the near-infrared. Conversely, III-V semiconductors are of particular interest in this context for their non-centrosymmetric nature. Among III-V zincblende candidates for efficient $\chi^{(2)}$ interactions, gallium arsenide (GaAs) possibly alloyed with $\mathrm{Al}$ offers the largest second-order susceptibility $\left(\chi_{\mathrm{xyz}}^{(2)} \simeq 200 \mathrm{pm} / \mathrm{V}\right)$ [8]. This explains why the first whispering-gallery-mode (WGM) secondharmonic generation at the micro-scale was demonstrated a few years ago with GaAs [9] and AlGaAs [10]. While in those cases the pump (second harmonic) was evanescently input (collected) into (from) a suspended microdisk via a tapered optical fiber, subsequent developments of semiconductor-on-insulator platforms based on wafer bonding have resulted in the integration of bus waveguides to WGM microresonators in different materials like GaN [11] and AlN [12, 13]. Despite their lower nonlinearity $\left(\chi_{\mathrm{GaN}}^{(2)} \simeq 20 \mathrm{pm} / \mathrm{V}\right.$ and $\left.\chi_{\mathrm{AlN}}^{(2)} \simeq 5 \mathrm{pm} / \mathrm{V}\right)$, these wide-gap semiconductors have enabled much higher SHG efficiencies at telecom wavelengths $\left(\eta_{\mathrm{SHG}} \simeq 14 \% \mathrm{~W}^{-1}\right.$ for $\mathrm{GaN}$ and $\eta_{\mathrm{SHG}} \simeq 165 \% \mathrm{~W}^{-1}$ for AlN). In particular, the latter performance resulted from quality factors higher than $10^{5}$ at both pump and $\mathrm{SH}$. With similar quality factors, an impressive $\eta_{\mathrm{SHG}} \simeq 65000 \%$ $\mathrm{W}^{-1}$ has been achieved with a GaAs-on-insulator microring [14] at the pump wavelength $\lambda_{\omega} \simeq 2 \mu \mathrm{m}$, which compares very favorably with respect to $\eta_{\mathrm{SHG}} \simeq 5 \% \mathrm{~W}^{-1}$ obtained at the same wavelength in Ref. [9].

However, the superior figures for these microrings are not inherent to the semiconductor-on-insulator technology, insofar Qfactors higher than $10^{6}$ have been reported for ALD-passivated suspended GaAs microdisks [15]. Additionally, suspended resonators are known to host pronounced optomechanical effects, as demonstrated both in GaAs [16] and AlGaAs [17]. As several works pointed out the beneficial scientifc impact of merging nonlinear $\chi^{(2)}$ and optomechanical interactions in the same resonator $[18,19]$, we are motivated to explore the integration of bus waveguides to a suspended AlGaAs microdisk. Indeed the absence of two-photon absorption in AlGaAs would enable operation at the telecom wavelength, while the resulting chip would be compatible with in-situ sensing applications and low temperature operation. Hence we report here on the design, fabrication, linear and nonlinear optical characterization of a suspended AlGaAs microdisk-based photonic circuit. Our platform enables us to demonstrate both second-harmonic generation (SHG) and spontaneous down-conversion (SPDC), providing the first steps towards an on-chip source of quantum light that could accommodate optomechanical effects.

Design. As can be seen in Fig. 1, three-wave mixing takes place in the microdisk located at the center of design, which carry whispering-gallery modes (WGM), at the wavelengths $\lambda_{\omega} \simeq 1600 \mathrm{~nm}$ and $\lambda_{2 \omega}=\lambda_{\omega} / 2 \simeq 800 \mathrm{~nm}$. We consider AlGaAs with an $\mathrm{Al}$ fraction above $28 \%$, hence not GaAs, in order to be able to perform SHG with a pump at telecom wavelengths and prevent the up-converted photon energy to exceed the material bandgap. This disk is evanescently coupled to two suspended 
waveguides that are set on its two sides, one designed at $\lambda_{\omega}$ and the other at $\lambda_{2 \omega}$. The light injection and collection is performed by approaching microlensed fibers to the extremities of the suspended waveguides using micropositioners, and piezo controllers for fine adjustement.

The microdisk is designed to achieve QPM between two WGMs. A growth of AlGaAs along the [001] direction indeed allows an azimuthal modulation of the effective second-order nonlinear susceptibility thanks to the $\overline{4}$ crystal symmetry, without resorting to domain inversion [9, 10, 20]: specifically, $\chi_{\mathrm{eff}}^{(2)}=$ $\chi_{\mathrm{xyz}}^{(2)} \sin (2 \varphi)$, with $\varphi$ the angle between the light propagation direction and the direction [100]. This condition is achieved if the azimuthal mode numbers $m_{\omega}$ and $m_{2 \omega}$ of the WGMs at $\lambda_{\omega}$ and $\lambda_{2 \omega}$, respectively, fulfill the relation $\Delta m=2 m_{\omega}-m_{2 \omega} \pm 2=0$. In thin AlGaAs epitaxy, this is made possible by coupling WGMs of radial mode numbers $p_{\omega}=1$ and $p_{2 \omega}=2$, and axial mode numbers $l_{\omega}=l_{2 \omega}=1$. Following the work of Ref. [10], a numerical simulation enables us to design the QPM between the TE-polarized $m_{\omega}=12 \mathrm{WGM}$ at $\lambda_{\omega}=1616 \mathrm{~nm}$ and the TMpolarized $m_{2 \omega}=26 \mathrm{WGM}$ at $\lambda_{2 \omega}=808 \mathrm{~nm}$, for an $\mathrm{Al}_{0.4} \mathrm{Ga}_{0.6} \mathrm{As}$ disk of thickness $h=155 \mathrm{~nm}$ and radius $R=1980 \mathrm{~nm}$.

The nonlinear performances of our devices are assessed by considering the SHG conversion efficiency, which can be written as a function of the linear transmissions at $\omega$ and $2 \omega\left(\eta_{\omega}\right.$ and $\left.\eta_{2} \omega\right)$ and a phase-matching contribution $\left(\eta_{\mathrm{PM}}\right)$ as: $\eta_{\mathrm{SHG}}\left(\lambda_{\omega}\right) \propto$ $\eta_{\omega}^{2}\left(\lambda_{\omega}\right) \eta_{2 \omega}\left(\lambda_{\omega}\right) \eta_{\mathrm{PM}}\left(\lambda_{\omega}\right)[21]$, where

$$
\begin{aligned}
\eta_{\omega}\left(\lambda_{\omega}\right) & =\frac{Q_{\omega} /\left(\pi m_{\omega}\right)}{1+\left(2 Q_{\omega}\left(\lambda_{\omega}-\lambda_{\omega}^{o}\right) / \lambda_{\omega}\right)^{2}} \\
\eta_{2 \omega}\left(\lambda_{\omega}\right) & =\frac{Q_{2 \omega} /\left(\pi m_{2 \omega}\right)}{1+\left(2 Q_{2 \omega}\left(\lambda_{\omega}-2 \lambda_{2 \omega}^{o}\right) / \lambda_{\omega}\right)^{2}} \\
\eta_{\mathrm{PM}}\left(\lambda_{\omega}\right) & \propto \operatorname{sinc}^{2}\left(\pi \Delta m\left(\lambda_{\omega}^{\mathrm{PM}}=2 \lambda_{2 \omega}^{\mathrm{PM}}\right)\right)
\end{aligned}
$$

with $\lambda_{\omega, 2 \omega}^{o}$ and $Q_{\omega, 2 \omega}$ the resonance wavelengths and quality factors of the disk modes, and $\lambda_{\omega, 2 \omega}^{\mathrm{PM}}$ the phase-matching wavelengths. Typical measured $Q$ factors are $Q_{\omega} \simeq 1.5 \times 10^{4}$ and $Q_{2 \omega} \simeq 1.5 \times 10^{3}$, and the spectral FWHM associated to $\eta_{\mathrm{PM}}$ is calculated to be close to $15 \mathrm{~nm}$. It is straightforward to notice that maximizing the nonlinear conversion efficiency implies to match the resonance and phase-matching wavelengths as follows: $\lambda_{\omega}^{o}=2 \lambda_{2 \omega}^{o}=\lambda_{\omega}^{\mathrm{PM}}=2 \lambda_{2 \omega}^{\mathrm{PM}}$. Fine tuning of these wavelengths is achieved by adjustment of the microdisk radius. At this stage, note that acting on one degree of freedom is sufficient as it allows to fulfill $\lambda_{\omega}^{o}=2 \lambda_{2 \omega}^{o}$ for the resonance associated with the $m_{\omega}$ and $m_{2 \omega}$ azimuthal numbers, therefore implying $\lambda_{\omega(2 \omega)}^{o}=\lambda_{\omega(2 \omega)}^{\mathrm{PM}}$. Based on the resonance matching condition, i.e. $\Delta \lambda=\lambda_{\omega}^{o}-2 \lambda_{2 \omega}^{o} \leq \lambda_{2 \omega}^{o} / 2 Q_{2 \omega}+\lambda_{\omega}^{o} / 2 Q_{\omega}=0.5 \mathrm{~nm}$, and on the quantity $\partial \Delta \lambda / \partial R \simeq 0.2$ (derived from the measurements of the resonance frequencies in disks of different radii), we find that a precision of $\pm 2.5 \mathrm{~nm}$ on the radius is required, which lies beyond the sample to sample reproducibility of our fabrication process of $\pm 20 \mathrm{~nm}$. To overcome this problem, we fabricate series of disks with step size of $5 \mathrm{~nm}$ on the radius.

The waveguides at $\lambda_{\omega}$ and $\lambda_{2 \omega}$ have been optimized by finiteelement simulations. Around the microdisk, the widths of the waveguides are tuned for coherent coupling at their respective wavelengths. Likewise, the gaps between the waveguides and the disk are designed to reach the critical coupling condition with equal intrinsic and coupling $Q$ factors, i.e. $Q_{i}=Q_{c}$, which maximizes the nonlinear conversion efficiency [9]. It also filters
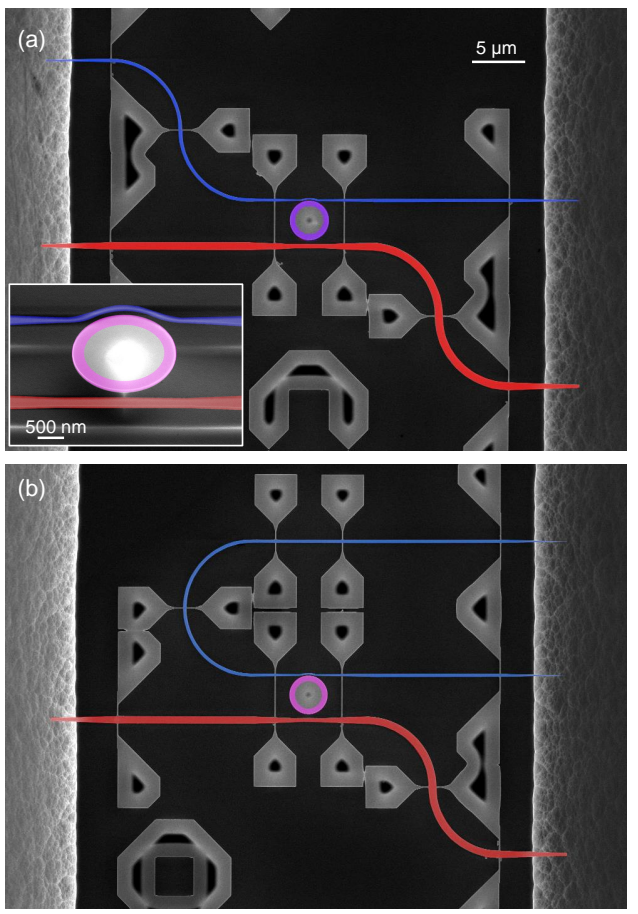

Fig. 1. SEM images of our photonic circuit: (a) configuration for linear measurements (zoom on the microdisk in inset), and (b) configuration for SHG and SPDC. The waveguides at $\lambda_{\omega}$ and $\lambda_{2 \omega}$ are colored in red and blue, respectively. They are not straight in order to reduce the unwanted coupling from one microlensed fiber to the other through free space. The rim of the microdisk where the three-wave mixing processes take place is pictured in violet.

the pump photons through destructive interference in the outgoing bus waveguide between the transmitted field and the field exiting the microdisk [22]. During preliminary tests, we have measured intrinsic $Q$ factors for WGMs at $\lambda_{\omega}$ and $\lambda_{2 \omega}$ using undercoupled waveguides: $Q_{i, \omega} \simeq 3 \times 10^{4}$ and $Q_{i, 2 \omega} \simeq 3 \times 10^{3}$. To match extrinsic $Q$ factors with these intrinsic ones, we use a $450 \mathrm{~nm}$-wide straight suspended waveguide at $\lambda_{\omega}$ distant from the rim of the disk by $400 \mathrm{~nm}$, and a $140 \mathrm{~nm}$-wide curved suspended waveguide at $\lambda_{2} \omega$ distant from the disk rim by $150 \mathrm{~nm}$ over $60^{\circ}$. Fine tuning of these parameters needs to be performed carefully in order to minimize the coupling between the WGM at $\lambda_{\omega}\left(\lambda_{2 \omega}\right)$ and the waveguide at $\lambda_{2 \omega}\left(\lambda_{\omega}\right)$.

In order to reduce losses at the anchoring points, the widths of the waveguides are enlarged as much as possible away from the disk in a way that preserves the single mode propagation. Anchoring is implemented using $100 \mathrm{~nm}$-wide tethers on both side of the waveguides. At their ends, the waveguides are narrowed until their effective index is about 1.2, enabling the mode to expand and thereby increasing the coupling efficiency with the microlensed fibers. The slopes of the tapered region of the waveguides next to the disk and at their extremities have been design for adiabatic single mode transmission of the signals. The whole structure sits on a $100 \mu \mathrm{m}$-tall mesa, allowing to approach the microlensed fibers in front of the tips of the inverted tapers.

Fabrication. A 155-nm-thick $\mathrm{Al}_{0.4} \mathrm{Ga}_{0.6}$ As layer is grown by molecular beam epitaxy on a GaAs substrate. The circuits are patterned by electronic lithography on Man2401 resist and then transferred to the AlGaAs layer by inductively coupled plasma 
etching in a $\mathrm{SiCl}_{4} / \mathrm{Ar}$ plasma. After this step, a wet selective isotropic etching of $\mathrm{GaAs}$ using the AlGaAs layer as a mask is done to define pedestals below the microdisks and to release the suspended waveguides. This etching is done in a $\mathrm{H}_{2} \mathrm{O}_{2}$ solution brought to $\mathrm{pH}=7$ by a few drops of ammonia solution. The mesa is then etch in $\mathrm{H}_{3} \mathrm{PO}_{4} / \mathrm{H}_{2} \mathrm{O}_{2} / \mathrm{H}_{2} \mathrm{O}: 1 / 1 / 1$ solution with a $\mathrm{S} 1828$ resist mask pattern by optical lithography.

Characterization. An external cavity diode laser and a Ti:Sapphire laser, both tunable and CW, are used to pump our device at $\lambda_{\omega}$ and $\lambda_{2 \omega}$, respectively. The detection is performed with InGaAs and Si photodiodes. Both sources and detectors can be connected to the sample through microlensed fibers. Probing linear transmissions at $\lambda_{\omega}$ and $\lambda_{2 \omega}$, SHG or SPDC signals involve coupling the relevant waveguides to the laser source on one side and to the detector on the other side. The polarizations are selected with fiber polarization controllers.

Linear transmissions. Away from resonances, the overall power transmission ratio of the microlensed fibers and AlGaAs suspended waveguides is $\simeq 30 \%$ for the $\lambda_{\omega}$-waveguide at $1600 \mathrm{~nm}$ and $\simeq 0.3 \%$ for the $\lambda_{2 \omega}$-waveguide at $800 \mathrm{~nm}$. The linear losses of the bare AlGaAs waveguides have been studied by manufacturing waveguides with facet terminations instead of inverted tapers and measuring the transmission spectra of these effective Fabry-Perot cavities [23]. For both waveguides, the high contrast of these oscillations points out a propagation loss close to $10 \%$, indicating that overall transmission of tapered waveguides is mainly limited by injection loss. We find coupling coefficients $T_{\mathcal{C}}$ between microlensed fibers and inverted tapers $T_{\mathcal{c}, \omega} \simeq 58 \%$ and $T_{c, 2 \omega} \simeq 6 \%$, in agreement with [24].

Fig. 2(a) shows the linear transmission spectra at $\lambda_{\omega}$ (red) and $\lambda_{2 \omega}$ (blue) for two devices of different radii: $R=1975 \mathrm{~nm}$ (left frame) with resonant wavelengths that do not match $\left(\lambda_{\omega}^{o} \neq\right.$ $2 \lambda_{2 \omega}^{o}$ ) and $R=1983 \mathrm{~nm}$ (right frame) with matching resonant wavelengths $\left(\lambda_{\omega}^{o}=2 \lambda_{2 \omega}^{o}\right)$. The WGMs can be identified by comparing their wavelengths and free spectral ranges with simulations. The experimental intensity contrasts of these resonances are not strictly equal to one as critical coupling would require, but are above $90 \%$ at $\lambda_{\omega}$ and $70 \%$ and $\lambda_{2 \omega}$. Fig. 2(b) gives the resonant wavelengths $\lambda_{\omega}^{o}$ and $\lambda_{2 \omega}^{o}$ of a series of microdisks as a function of their radii, and linear fits. The crossing of the two plots indeed shows that double resonance occurs for a radius close to $1983 \mathrm{~nm}$.

Second-harmonic generation. SHG has been performed on these different devices at $\lambda_{\omega}^{\mathrm{PM}} \simeq 1620 \mathrm{~nm}$. The signal is collected on a Si photodiode through the $\lambda_{2 \omega}$-waveguide and fibers, all these elements efficiently filtering the pump. Fig. 2(a) shows SHG conversion efficiency spectra (in green, right-hand side $y$-axis) for the two above situations of unmatching and matching resonances. The position of the maxima and the linewidths of the SHG conversion efficiency spectra correspond to those of the transmissions at $\lambda_{\omega}$, as can be expected from this sum-frequency process. In the doubly resonant case, the SHG conversion efficiency amounts to $5 \% \mathrm{~W}^{-1}$, i.e. around two orders of magnitude higher than the unmatched case, and is maximum over the series of disk measured as can be seen from Fig. 2(c). This value has been reached for $\lambda_{\omega}=1622.6 \mathrm{~nm}$, with input and output powers in the waveguides of $116 \mu \mathrm{W}$ and $0.66 \mathrm{nW}$, respectively. The $\mathrm{x}$-axis of this figure has been estimated from the knowledge of the resonance frequencies, according to the fits $\lambda_{\omega}(R)$ of Fig. 2(b). Following Ref. [20], we performed a theoretical calculation of the SHG conversion efficiency of this device and find an expected value of $15 \% \mathrm{~W}^{-1}$, in good agreement with
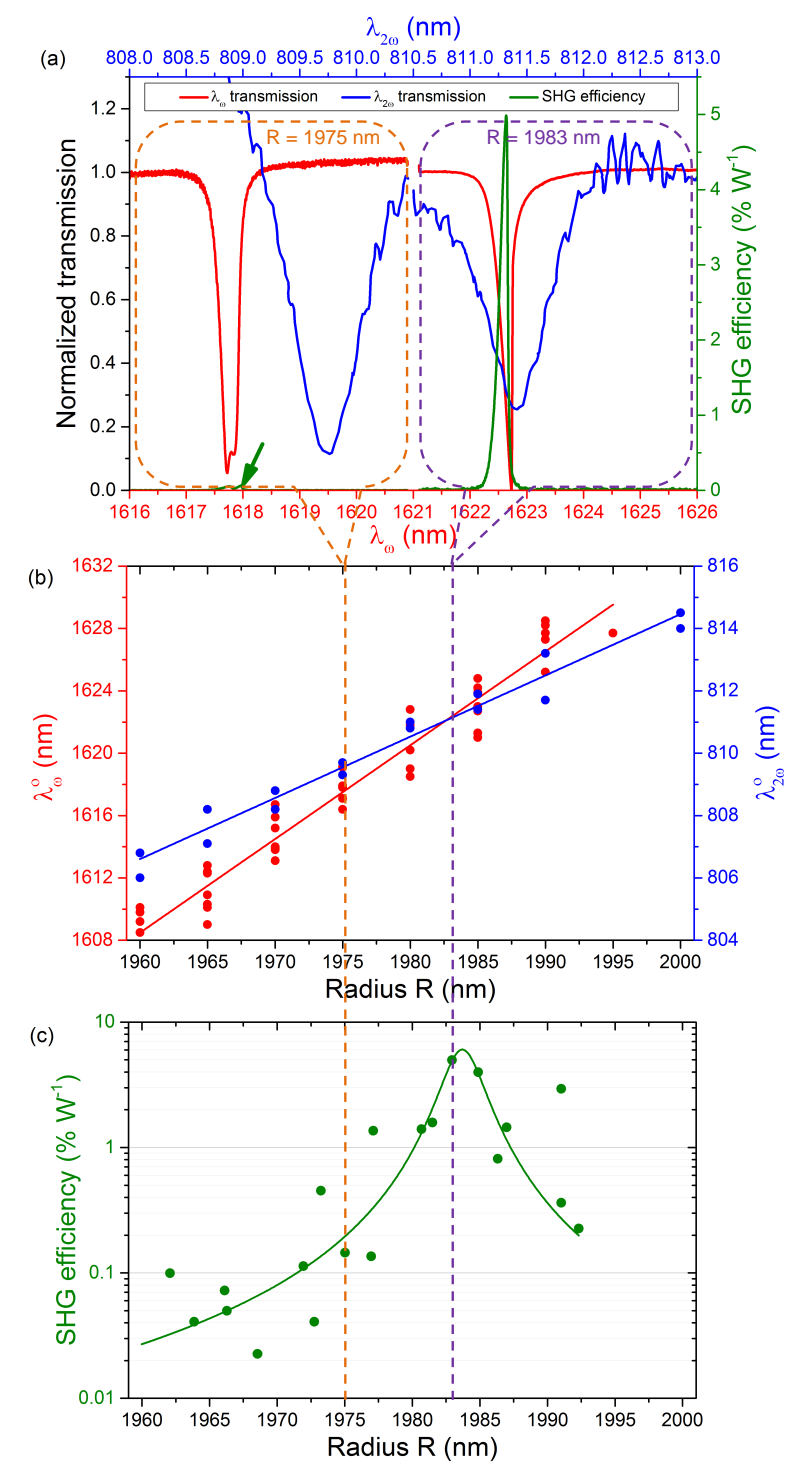

Fig. 2. Characterization of a series of microdisks with different radii. (a) Experimental spectra of linear transmissions at $\lambda_{\omega}$ (red) and $\lambda_{2 \omega}$ (blue), and SHG efficiency (green) for two different disks: with unmatching (left) and matching (right) resonances. (b) Resonant wavelengths $\lambda_{\omega}^{o}$ and $\lambda_{2 \omega^{\prime}}^{o}$ and (c) SHG efficiency as a function of the microdisk radius. Experimental data in circles and fits in solid lines, linear in (b) and Lorentzian in (c).

the experimental results. It has also been checked that SHG is selective to the pump polarization and that the $\mathrm{SH}$ power scales quadratically with the input pump power. Compared with previous measurements performed on $\mathrm{AlGaAs}$ microdisks, in the important telecom range, it exceeds by a factor 150 that of Ref. [10] $\left(7 \times 10^{-4} \mathrm{~W}^{-1}\right)$.

Spontaneous down-conversion. We have used the device that gave the best SHG performances (i.e. under the double resonance condition, $R=1983 \mathrm{~nm}$ ) to perform a SPDC measurement, not yet reported with (Al)GaAs microdisks. The source is here modulated in intensity using an acousto-optic modulator to produce pulses of duration $500 \mathrm{~ns}$ at a repetition rate $20 \mathrm{kHz}$ in order to reach higher down-converted energies through higher input peak powers, while keeping the device below its damage 

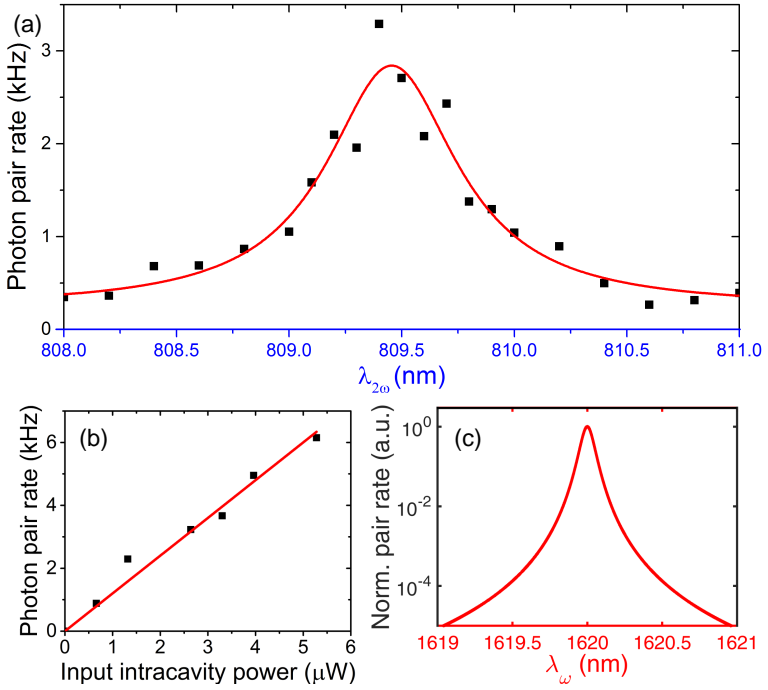

Fig. 3. (a) Acceptance spectrum: SPDC photon pair rate vs. pump wavelength $\lambda_{2 \omega}$ in a doubly resonant disk and for $1.8 \mu \mathrm{W}$ mean intracavity pump power, and analytical calculation (solid line). (b) Behaviour with respect to pump intracavity power at $\lambda_{2 \omega}^{o}$. (c) Analytically calculated spectral distribution of one photon of the generated pairs, for $\lambda_{2 \omega}^{o}$.

threshold $\left(\simeq 500 \mu \mathrm{W}\right.$ mean power at $\lambda_{2 \omega}$, and $10 \mathrm{~mW}$ at $\left.\lambda_{\omega}\right)$. The down-converted photons at $\lambda_{\omega}$ are detected with a singlephoton InGaAs/InP avalanche photodiode, and the pump photons at $\lambda_{2 \omega} \simeq 810 \mathrm{~nm}$ are filtered with $1200 \mathrm{~nm}$-low-pass filters.

Fig. 3(a) shows the measured SPDC acceptance spectrum, i.e. is performed by sweeping the pump frequency $\lambda_{2 \omega}$, for a pump mean intracavity power of $1.8 \mu \mathrm{W}$. The maximal experimental biphoton generation rate reaches $3 \mathrm{kHz}$, which is again in good agreement with the expected rate that can be inferred from the measured SHG conversion efficiency $\eta_{\mathrm{SPDC}}^{\text {th }}=\eta_{\mathrm{SHG}} \frac{\hbar \omega}{4 \pi} \frac{\omega}{\mathrm{Q}_{2 \omega}}[25]$, and amounts to $13 \mathrm{kHz}\left(\eta_{\mathrm{SPDC}}^{\text {th }}=8.3 \times 10^{-10}\right)$. The linear dependence of this rate with the input intracavity power is shown in Fig. 3(b) for $\lambda_{2 \omega}^{o}$. Additionally, the photon pair creation rate is maximized (minimized) when the pump polarization is TM (TE), as expected.

Although the down-converted photons have not been resolved in frequency, we calculated their spectrum to be narrowband (0.7 nm FWHM) in the doubly resonant configuration, as shown in Fig. 3(c). By reaching higher $Q$ factors, hence narrower bandwidths, this device would perform as a source of two indistinguishable photons, and of even Schrödinger kitten states in the low photon number regime. Conversely, if the pump is detuned from its central resonance frequency (by $\lesssim \lambda_{2 \omega}^{o} / 2 Q_{2 \omega}$ ) or if the two resonances do not match, the two non-degenerated down-converted photons are entangled in frequency and still expected to be efficiently produced, leaving only one of them on resonance at $\lambda_{\omega}$. This explains the bandwidth of the measured SPDC acceptance spectrum (as large as that of the resonance at $\left.\lambda_{2 \omega}\right)$, which is well described by the calculation based on $\eta_{\mathrm{SHG}}$ and $\eta_{\text {SPDC }}$, shown as the solid line of Fig. 3(a). It is also consistent with the experimental observation that the SPDC efficiency do not vary significantly when $\lambda_{\omega}^{o} \neq 2 \lambda_{2 \omega}^{o}$. Combined with a dichroic mirror and in a low photon number regime, the device could then act as a heralded source of single photons.

Conclusion. We designed and realized a four-port AlGaAs pho- tonic chip based on a suspended doubly resonant microdisk supporting WGMs. This fully integrable and robust device enabled us to demonstrate efficient SHG and SPDC. It is attractive both for frequency conversion, and for the generation of separable or entangled biphoton states (whether it is operated to perform as degenerate or non-degenerate SPDC) for quantum applications in the telecom range. This work could be extended to allow spectral tuning of the generated photons, e.g. by means of temperature or voltage control, and is already compatible with the implementation of optomechanical effects, whose combinaison with $\chi^{(2)}$ quantum photonics remains to be explored.

Funding. ASTRID Project SPAIN (ANR-18-ASTR-0007).

Disclosures. The authors declare no conflicts of interest.

\section{REFERENCES}

1. D. Armani, T. Kippenberg, S. Spillane, and K. Vahala, Nature 421, 925 (2003).

2. S. McCall, A. Levi, R. Slusher, S. Pearton, and R. Logan, Appl. Phys. Lett. 60, 289 (1992).

3. D. Vernooy, A. Furusawa, N. P. Georgiades, V. Ilchenko, and H. Kimble, Phys. Rev. A 57, R2293 (1998).

4. T. Kippenberg, H. Rokhsari, T. Carmon, A. Scherer, and K. Vahala, Phys. Rev. Lett. 95, 033901 (2005).

5. E. Gil-Santos, C. Baker, D. Nguyen, W. Hease, C. Gomez, A. Lemaître, S. Ducci, G. Leo, and I. Favero, Nat. Nanotechnol. 10, 810 (2015).

6. K. Djordjev, S.-J. Choi, S.-J. Choi, and R. Dapkus, IEEE Photonics Technol. Lett. 14, 828 (2002).

7. D. V. Strekalov, A. S. Kowligy, Y.-P. Huang, and P. Kumar, New J. Phys. 16, 053025 (2014).

8. I. Shoji, T. Kondo, A. Kitamoto, M. Shirane, and R. Ito, JOSA B 14, 2268 (1997).

9. P. S. Kuo, J. Bravo-Abad, and G. S. Solomon, Nat. Comm. 5, 3109 (2014).

10. S. Mariani, A. Andronico, A. Lemaître, I. Favero, S. Ducci, and G. Leo, Opt. Lett. 39, 3062 (2014).

11. I. Roland, M. Gromovyi, Y. Zeng, M. El Kurdi, S. Sauvage, C. Brimont, T. Guillet, B. Gayral, F. Semond, J.-Y. Duboz, M. de Micheli, X. Checoury, and P. Boucaud, Sci. Reports 6, 34191 (2016).

12. X. Guo, C.-I. Zou, C. Schuck, H. Jung, R. Cheng, and H. X. Tang, Light. Sci. \& Appl. 6, e16249 (2017).

13. A. W. Bruch, X. Liu, J. B. Surya, C.-L. Zou, and H. X. Tang, Optica. 6, 1361 (2019).

14. L. Chang, A. Boes, P. Pintus, J. D. Peters, M. Kennedy, X.-W. Guo, N. Volet, S.-P. Yu, S. B. Papp, and J. E. Bowers, APL Photonics 4, 036103 (2019).

15. B. Guha, F. Marsault, F. Cadiz, L. Morgenroth, V. Ulin, V. Berkovitz, A. Lemaître, C. Gomez, A. Amo, S. Combrié, B. Gérard, G. Leo, and I. Favero, Optica 4, 218 (2017).

16. L. Ding, C. Baker, P. Senellart, A. Lemaitre, S. Ducci, G. Leo, and I. Favero, Phys. Rev. Lett. 105, 263903 (2010).

17. B. Guha, S. Mariani, A. Lemaître, S. Combrié, G. Leo, and I. Favero, Opt. Express 25, 24639 (2017).

18. S. Huang and G. Agarwal, Phys. Rev. A 79, 013821 (2009).

19. V. Peano, H. Schwefel, C. Marquardt, and F. Marquardt, Phys. Rev. Lett. 115, 243603 (2015).

20. Y. Dumeige and P. Feron, Phys. Rev. A 74, 063804 (2006).

21. P. S. Kuo and G. S. Solomon, Opt. Express 19, 16898 (2011).

22. A. Yariv, Electron. Lett. 36, 321 (2000).

23. A. De Rossi, V. Ortiz, M. Calligaro, L. Lanco, S. Ducci, V. Berger, and I. Sagnes, J. Appl. Phys. 97, 073105 (2005).

24. N. Morais, I. Roland, M. Ravaro, W. Hease, A. Lemaître, C. Gomez, S. Wabnitz, M. De Rosa, I. Favero, and G. Leo, Opt. Lett. 42, 4287 (2017).

25. A. Yariv, Quantum Electronics (3rd ed.) John Wiley \& Sons (1989). 


\section{FULL REFERENCES}

1. D. Armani, T. Kippenberg, S. Spillane, and K. Vahala, "Ultra-high-q toroid microcavity on a chip," Nature. 421, 925 (2003).

2. S. McCall, A. Levi, R. Slusher, S. Pearton, and R. Logan, "Whisperinggallery mode microdisk lasers," Appl. Phys. Lett. 60, 289-291 (1992).

3. D. Vernooy, A. Furusawa, N. P. Georgiades, V. Ilchenko, and H. Kimble, "Cavity qed with high-q whispering gallery modes," Phys. Rev. A 57, R2293 (1998).

4. T. Kippenberg, H. Rokhsari, T. Carmon, A. Scherer, and K. Vahala, "Analysis of radiation-pressure induced mechanical oscillation of an optical microcavity," Phys. Rev. Lett. 95, 033901 (2005).

5. E. Gil-Santos, C. Baker, D. Nguyen, W. Hease, C. Gomez, A. Lemaître, S. Ducci, G. Leo, and I. Favero, "High-frequency nano-optomechanical disk resonators in liquids," Nat. Nanotechnol. 10, 810 (2015).

6. K. Djordjev, S.-J. Choi, S.-J. Choi, and R. Dapkus, "Microdisk tunable resonant filters and switches," IEEE Photonics Technol. Lett. 14, 828830 (2002).

7. D. V. Strekalov, A. S. Kowligy, Y.-P. Huang, and P. Kumar, "Optical sumfrequency generation in a whispering-gallery-mode resonator," New $\mathrm{J}$. Phys. 16, 053025 (2014).

8. I. Shoji, T. Kondo, A. Kitamoto, M. Shirane, and R. Ito, "Absolute scale of second-order nonlinear-optical coefficients," JOSA B 14, 2268-2294 (1997).

9. P. S. Kuo, J. Bravo-Abad, and G. S. Solomon, "Second-harmonic generation using-quasi-phasematching in a gaas whispering-gallerymode microcavity," Nat. Commun. 5, 3109 (2014).

10. S. Mariani, A. Andronico, A. Lemaître, I. Favero, S. Ducci, and G. Leo, "Second-harmonic generation in algaas microdisks in the telecom range," Opt. Lett. 39, 3062-3065 (2014).

11. I. Roland, M. Gromovyi, Y. Zeng, M. El Kurdi, S. Sauvage, C. Brimont, T. Guillet, B. Gayral, F. Semond, J.-Y. Duboz, M. de Micheli, $X$. Checoury, and P. Boucaud, "Phase-matched second harmonic generation with on-chip gan-on-si microdisks," Sci. Reports 6, 34191 (2016).

12. X. Guo, C.-I. Zou, C. Schuck, H. Jung, R. Cheng, and H. X. Tang, "Parametric down-conversion photon-pair source on a nanophotonic chip," Light. Sci. \& Appl. 6, e16249 (2017).

13. A. W. Bruch, X. Liu, J. B. Surya, C.-L. Zou, and H. X. Tang, "Onchip $\chi^{(2)}$ microring optical parametric oscillator," Optica. 6, 1361-1366 (2019).

14. L. Chang, A. Boes, P. Pintus, J. D. Peters, M. Kennedy, X.-W. Guo, N. Volet, S.-P. Yu, S. B. Papp, and J. E. Bowers, "Strong frequency conversion in heterogeneously integrated gaas resonators," APL Photonics 4, 036103 (2019).

15. B. Guha, F. Marsault, F. Cadiz, L. Morgenroth, V. Ulin, V. Berkovitz, A. Lemaître, C. Gomez, A. Amo, S. Combrié, B. Gérard, G. Leo, and I. Favero, "Surface-enhanced gallium arsenide photonic resonator with quality factor of 6× 10 6," Optica 4, 218-221 (2017).

16. L. Ding, C. Baker, P. Senellart, A. Lemaitre, S. Ducci, G. Leo, and I. Favero, "High frequency gaas nano-optomechanical disk resonator," Phys. Rev. Lett. 105, 263903 (2010).

17. B. Guha, S. Mariani, A. Lemaître, S. Combrié, G. Leo, and I. Favero, "High frequency optomechanical disk resonators in iii-v ternary semiconductors," Opt. Express 25, 24639-24649 (2017).

18. S. Huang and G. Agarwal, "Enhancement of cavity cooling of a micromechanical mirror using parametric interactions," Phys. Rev. A 79, 013821 (2009).

19. V. Peano, H. Schwefel, C. Marquardt, and F. Marquardt, "Intracavity squeezing can enhance quantum-limited optomechanical position detection through deamplification," Phys. Rev. Lett. 115, 243603 (2015).

20. Y. Dumeige and P. Feron, "Whispering-gallery-mode analysis of phasematched doubly resonant second-harmonic generation," Phys. Rev. A 74, 063804 (2006).

21. P. S. Kuo and G. S. Solomon, "On-and off-resonance second-harmonic generation in gaas microdisks," Opt. Express 19, 16898-16918 (2011).

22. A. Yariv, "Universal relations for coupling of optical power between microresonators and dielectric waveguides," Electron. Lett. 36, 321322 (2000).
23. A. De Rossi, V. Ortiz, M. Calligaro, L. Lanco, S. Ducci, V. Berger, and I. Sagnes, "Measuring propagation loss in a multimode semiconductor waveguide," J. Appl. Phys. 97, 073105 (2005).

24. N. Morais, I. Roland, M. Ravaro, W. Hease, A. Lemaître, C. Gomez, S. Wabnitz, M. De Rosa, I. Favero, and G. Leo, "Directionally induced quasi-phase matching in homogeneous algaas waveguides," Opt. Lett. 42, 4287-4290 (2017).

25. A. Yariv, Quantum Electronics (3rd ed.) John Wiley \& Sons (1989). 